\title{
Gestión de riesgos operacionales en cadenas de suministro agroalimentarias bajo un enfoque de manufactura esbelta
}

\author{
Andrés M. Paredes-Rodriguez ${ }^{1}$, Vivian L. Chud-Pantoja ${ }^{2^{*}}$ y Claudia C. Peña-Montoya ${ }^{3}$ \\ (1) Facultad de Ingeniería, Programa de Ingeniería Industrial, Corporación Universitaria Minuto de Dios, \\ Buga - Colombia (correo-e: aparedesrod@uniminuto.edu.co) \\ (2) Ingeniería Industrial, Universidad del Valle, Sede Zarzal, Zarzal - Colombia \\ (correo-e: vivian.chud@correounivalle.edu.co) \\ (3) Facultad de Ingeniería, Departamento de Operaciones y Sistemas, Universidad Autónoma de Occidente, \\ Cali-Colombia. (correo-e: ccpena@uao.edu.co) \\ * Autor a quien debe ser dirigida la correspondencia.
}

Recibido Jul. 8, 2021; Aceptado Sep. 1, 2021; Versión final Oct. 30, 2021, Publicado Feb. 2022

\begin{abstract}
Resumen
El objetivo de este estudio es evaluar herramientas de manufactura esbelta como una estrategia de mitigación de riesgos operacionales en cadenas de suministro del aguacate Hass en el departamento del Valle del Cauca, Colombia. Se usa el mapa de la cadena de valor y se identifican los riesgos. Para priorizarlos, se implementa el Análisis Modal de Efectos y Fallas (AMFE), y el Despliegue de Función Calidad y Lógica Difusa (FQFD). Estos se relacionan con las mudas para proponer herramientas de acciones de mitigación asociadas a herramientas de manufactura esbelta. Los resultados muestran que las $5 S$ (Sort, Set-in-order, Shine, Standardize, Sustain), el Mantenimiento Productivo Total (TPM), el trabajo estándar y la gestión visual respaldan la mitigación de los riesgos en los eslabones estudiados. En conclusión, la cadena productiva está compuesta por productores, transporte primario, línea de empaque y transporte secundario, los cuales son eslabones fundamentales y la fuente principal de riesgos operacionales.
\end{abstract}

Palabras clave: gestión de riesgo operacional; cadenas de suministro; agroalimentario; manufactura esbelta; aguacate Hass; mapa de valor

\section{Operational risk management in agri-food supply chains under a lean manufacturing approach}

\begin{abstract}
The objective of this study is to assess lean manufacturing tool as a strategie that mitigate operational risks in the supply chains of Hass avocado in the state of Valle del Cauca, Colombia. Value stream mapping is applied and risks are characterized and prioritized by using FMEA (Failure Mode and Effect Analysis) and FQFD (Fuzzy Quality Function Deployment). Risks are correlated to waste to propose mitigation actions associated with lean manufacturing tools. The results show that $5 \mathrm{~S}$ (Sort, Set in order, Shine, Standardize, and Sustain), Total Productive Maintenance (TPM), standard work, and visual management support the mitigation of the risks examined. In conclusion, the productive chain is composed of producers, primary transport, packing lines, and secondary transport, which are essential links and the main sources of operational risks.
\end{abstract}

Keywords: operational risk management; supply chains; agri-food; lean manufacturing; Hass avocado; value stream mapping 


\section{INTRODUCCIÓN}

En la actualidad, las cadenas de abastecimiento se enfrentan a diversas problemáticas derivadas de los cortos ciclos de vida de los productos, la competencia más intensa y el aumento de las expectativas del cliente (Blome et al., 2014) lo que ha generado la necesidad urgente de cambiar la forma tradicional de gestionar las cadenas de suministro, con el propósito de aumentar la productividad de los procesos y reducir los desperdicios de tiempo, costo y espacio. Además, dadas las condiciones globales de las cadenas, se incrementan los riesgos de las mismas, es decir, los eventos que pueden interrumpir las condiciones normales de las actividades en los procesos operacionales, conduciendo a un impacto en el desempeño.

De esta manera, la gestión del riesgo de la cadena de suministro (SCRM, por sus siglas en inglés) es una actividad de gestión estratégica en las empresas, ya que puede afectar el desempeño operacional, de mercado y financiero (Narasimhan y Talluri, 2009). Así, la gestión eficaz del riesgo de la cadena de suministro (CS) es esencial para que un negocio sea exitoso y representa una competencia y capacidad que muchas empresas todavía tienen que desarrollar (Lavastre et al, 2012). Tang (2006) distingue dos tipos de riesgos asociados a cadenas de abastecimiento: operacionales y disruptivos. Los riesgos operacionales hacen relación al efecto que tiene la incertidumbre en los procesos cotidianos dentro de las compañías, por el contrario, los riesgos disruptivos son interrupciones causadas por agentes externos a la empresa como son los desastres naturales, crisis económicas y desastres ocasionados por el hombre. Esta investigación considerará los riesgos operacionales en una cadena de abastecimiento debido a que, a pesar de ser menos frecuentes, estos tienen un impacto negativo en los procesos que puede ampliarse a lo largo de toda la cadena, sino se atienden correcta y oportunamente (Osorio et al., 2017).

Si bien es cierto que distintos autores definen diferentes etapas para un proceso de gestión de riesgo operacional en cadenas de abastecimiento, en esta investigación se utilizará las tres primeras fases definidas por Osorio et al. (2017), las cuales incluyen: identificación, medición y evaluación, y gestión del riesgo, entendiendo esta última como una etapa de mitigación de riesgos. Para la determinación de los riesgos asociados a la cadena de suministro, se reconoce la herramienta de manufactura esbelta definida como Mapeo de la Cadena de Valor (VSM, por sus siglas en inglés), dado que permite caracterizar la cadena, analizar de manera detallada cada una de las etapas o procesos e identificar los aspectos que pueden eliminarse porque no agregan valor, lo que se le relaciona como desperdicios del proceso (De Steur et al., 2016). En este sentido, los riesgos generan interrupciones en las operaciones dentro de la cadena de suministro, que se convierten en actividades que no agregan valor y por lo tanto es necesario crear planes de acción que permita reducir estos eventos que generan desperdicios.

Mientras que la gestión del riesgo ha sido abordada en diferentes cadenas de suministro, existe una necesidad visible de mayor investigación en cadenas de suministro de productos perecederos y la presentación de estrategias sólidas para gestionar los riesgos, las incertidumbres y mejorar el rendimiento de estas (Prakash et al., 2017) resaltando una oportunidad de investigación en las cadenas de suministro de alimentos (Nakandala et al., 2016). Además, se sugiere investigar la gestión del riesgo en diferentes cadenas de suministro y desarrollar estrategias basadas en sus entornos (Venkatesh et al., 2015).

Las cadenas de suministro agrícolas se encuentran conformadas por todas las etapas de producción, procesamiento, comercio, distribución y consumo de alimentos frescos, las cuales se encargan de proporcionar la seguridad alimentaria de la población, al mismo tiempo que se garantiza el crecimiento y desarrollo económico del país. Hoy en día, estas cadenas de suministro se enfrentan a distintas problemáticas provenientes de la incertidumbre y variabilidad propia del entorno, los cuales las hacen vulnerables ante distintos riesgos en su operación (Zhao et al., 2020). En Colombia, una de las cadenas agrícolas con mayor proyección en el mercado es la cadena del aguacate Hass (Escobar et al., 2019); sin embargo, se ha identificado que este tipo de cadenas productivas presentan limitaciones con relación al cultivo, al sistema de producción, comercialización y manejo tecnológico (Méndez, 2016). Esto significa la presencia de posibles riesgos por fallas en las operaciones, con lo cual se reducen las posibilidades de convertirse en cadenas de suministro globales (Prakash et al., 2017).

Dado que la industria de aguacate en Colombia es reciente, se han encontrado pocas investigaciones e innovaciones sobre la misma. Debido a lo anterior se hace necesario estudiar las operaciones de los actores de la red con el objetivo de identificar los riesgos asociados y establecer estrategias de mitigación para los mismos, considerando la relación entre riesgos y desperdicios dado que ambos son considerados como elementos que afectan el buen desempeño de la cadena (Sodhi et al., 2012). Cabe resaltar que las distintas herramientas de manufactura esbelta representan una solución a la reducción de desperdicios (RiveraCadavid, 2011), y por tanto, un aporte al establecimiento de planes de acción que busquen mejorar la competitividad de la cadena (Cardozo et al., 2011), lo que se puede traducir en la minimización de los riesgos, porque buscan garantizar un manejo eficiente de los recursos y reducir los desperdicios encontrados (Rivera 
Cadavid, 2011). La implementación de herramientas de manufactura esbelta en cadenas agroalimentarias todavía está creciendo, no obstante, la herramienta VSM ha demostrado mejorar el desempeño de este tipo de cadenas, a través de la identificación de puntos críticos para la disminución de la pérdida de alimentos (De Steur et al., 2016). Por ejemplo, Wesana et al. (2019) utilizan la mapeo de cadena de valor como una estrategia para medir las pérdidas presentes en una cadena de suministro de lácteos en Uganda, con el propósito de identificar e intervenir aquellas actividades que provocaban un mayor índice de desperdicios de alimentos en la red.

Otros autores han aplicado VSM en cadenas agroalimentarias, con el fin de reducir los desperdicios, proponer mejoras en los procesos logísticos, determinar y eliminar las actividades que no agregan valor (DelgadoAlvarez et al, 2018). Asimismo, De Oliveira et al. (2020) implementa técnicas esbeltas en granjas verticales para definir mejoras que permitan aumentar el rendimiento. Es importante resaltar que los estudios analizados, no consideran el riesgo como agente generador de desperdicio dentro de los procesos de una cadena de abastecimiento, lo cual es un aporte de esta investigación, puesto que se analiza la relación existente entre riesgos y desperdicios para establecer estrategias esbeltas que mitiguen los riesgos.

En esta investigación se utiliza el VSM desde un enfoque exclusivamente de caracterización de las operaciones de los eslabones de la cadena de suministro del aguacate Hass (productores, transporte primario, línea de empaque y transporte secundario); con lo que se logra identificar los riesgos asociados a las operaciones de cada eslabón vistos como interrupciones en la cadena de suministro agroalimentaria, que generan desperdicios en la cadena y a partir de estos, se proponen alternativas de mitigación de riesgos, basadas en herramientas de manufactura esbelta, que reduzcan la ocurrencia de los riesgos y conlleven a la eliminación de actividades que no agregan valor.

\section{METODOLOGÍA}

La metodología propuesta y utilizada en esta investigación consta de cuatro fases, caracterización de la cadena de suministro, identificación, evaluación (priorización) y mitigación de los riesgos, tal como se observa en la Figura 1.

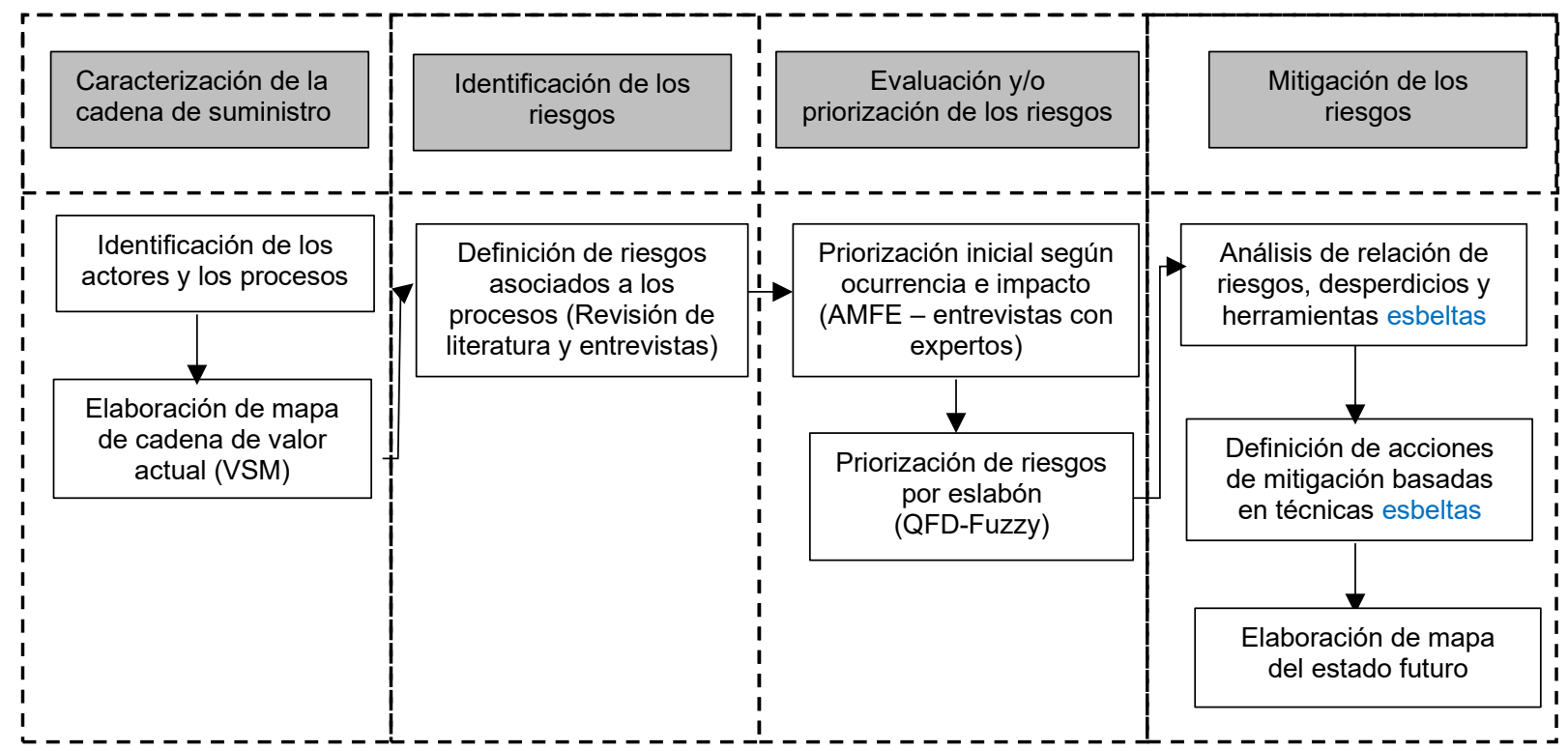

Fig. 1: Metodología para la propuesta de herramientas de manufactura esbelta como acciones de mitigación de riesgos operacionales.

\section{Fase 1: Caracterización de la cadena de suministro}

Se identificaron los actores que componen la cadena y aquellos que son representativos en las operaciones, para esto se utilizaron fuentes primarias como entrevistas a distintas organizaciones y representantes de compañías que se dedican a la producción, empaque y transporte de aguacate. Se llevaron a cabo entrevistas semiestructuradas (REF) a ocho (8) expertos que accedieron a desarrollar la entrevista, debido a las limitaciones que impuso el covid 19 durante al año 2020. Las preguntas estuvieron dirigidas a indagar aspectos generales, operacionales, gestión del riesgo y valoración de los riesgos. También se consultaron fuentes secundarias de los documentos oficiales relacionados con el sector publicados por el Instituto Colombiano Agropecuario (ICA, 2012). El levantamiento de información permitió identificar 4 eslabones principales. 
También se uso el Mapeo de la Cadena de Valor (VSM) como herramienta de manufactura esbelta para el diagnóstico de los procesos en los principales eslabones, de tal manera que se identificaron los desperdicios o actividades que no agregan valor y que son fuente de riesgos operacionales, es decir, que interrumpen las actividades y el funcionamiento normal de las mismas y se representaron en los mapas actuales de la cadena.

Fase 2: Identificación de los riesgos

Se realizó una revisión de literatura de los principales riesgos operacionales presentes en cadenas de suministro agroindustriales, posteriormente se analizaron aquellos que pueden presentarse en cada uno de los eslabones a partir de las respuestas obtenidas en las entrevistas, resultó un listado clasificado.

\section{Fase 3: Priorización de los riesgos}

Se priorizaron los riesgos operacionales por eslabón, considerando la probabilidad de ocurrencia y el impacto, mediante dos herramientas; Análisis Modal de Efectos y Fallas (AMFE) con la que se obtuvo un listado priorizado inicial y QFD difuso (Osorio et al. (2018)), con el que se priorizaron los riesgos según el impacto sobre los objetivos estratégicos de la cadena de suministro o eslabón. Para la aplicación de ambas herramientas se utilizaron encuestas a expertos del sector cuyo objetivo principal fue la valoración y juicio de la ocurrencia y del impacto de los riesgos bajo una escala Likert, donde 1 era muy baja ocurrencia o muy bajo impacto y 5 muy alta ocurrencia o muy alto impacto; también definían si aplicaba o no el riesgo para el eslabón correspondiente. Esta encuesta la contestaron 21 expertos del sector productor, 5 del transporte y 5 de línea de empaque.

\section{Fase 4: Mitigación de los riesgos}

En esta fase se definieron las estrategias de mitigación, una etapa fundamental de la gestión del riesgo en las cadenas de suministro, ya que representa la oportunidad de eliminar o mitigar el impacto que generan los riesgos en las actividades de la organización, y se espera que estas sean fácilmente realizables (Kırılmaz y Erol, 2017). Una vez obtenidos los riesgos priorizados por eslabón, se realizó una matriz de relación de riesgos, desperdicios y herramientas de manufactura esbelta relacionadas, la cual representa las estrategias de mitigación de riesgos. Posteriormente se analizaron las acciones de mitigación relacionadas con la aplicación de las herramientas de manufactura esbelta, según el VSM actual y se definieron cuáles riesgos son impactados, finalmente en un VSM de estado futuro se representó la relación entre operaciones, estrategias de mitigación, acciones de mitigación o eliminación de desperdicios y riesgos impactados

\section{RESULTADOS Y DISCUSIÓN}

Los resultados se presentan según las fases propuestas, fue aplicado en la cadena productiva de aguacate Hass en el departamento del Valle del Cauca, Colombia.

\section{Fase 1: Caracterización de la cadena de suministro}

La cadena de suministro del aguacate Hass en Colombia y particularmente en el departamento del Valle del Cauca, ha sido priorizada para el crecimiento de la región (CRC, 2015), los actores o eslabones que componen la cadena y el flujo de los productos se pueden observar en la Figura 2. Luego se presenta la definición de los eslabones que hacen parte de la cadena productiva del aguacate:

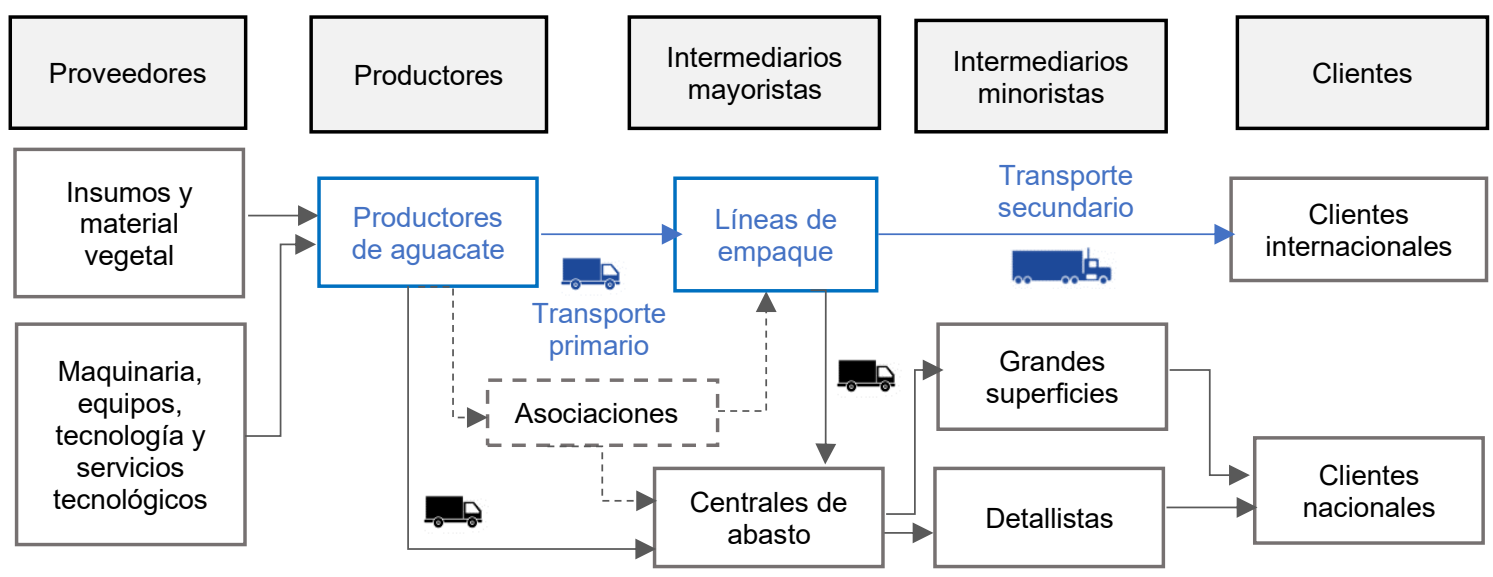

Fig. 2: Representación de la cadena de suministro del aguacate Hass en el departamento del Valle del Cauca 
Proveedores: Agrupa a los integrantes de la cadena que tienen que ver con el suministro de insumos agroquímicos y biológicos, otros insumos o servicios; materiales y herramientas; maquinaria, equipos y tecnología, necesarios para una producción competitiva (Mosquera Abadía et al., 2016).

Productores: Son los encargados del proceso de producción de un bien natural propio de la región, siendo este el caso del aguacate. Actualmente en el departamento existen unos lugares de producción habilitados por el Instituto Colombiano Agropecuario (ICA), las cuales son: La Guadalupe (Bolívar), Finca el Horizonte (Bolívar), La María 2 (Sevilla), La Libia (Trujillo) y el Jardín de Venus (Tuluá). Sin embargo, en el Valle del Cauca se cuenta con otros productores de aguacate, que no han sido habilitados por el ICA.

Líneas de empaque: Formado por acopiadores, comercializadores mayoristas y/o empresa de empaque de la fruta, son el puente entre el productor y el consumidor, tanto para el mercado nacional como internacional (Mosquera Abadía et al., 2016). Se encargan de transformar lo que produce la tierra y generan un aporte a los pequeños productores mediante acompañamiento para un desarrollo técnico que permita garantizar la calidad del aguacate, y realizar el empaquetado para la exportación, abriendo canales de comercialización internacional y dando cumplimiento a los estándares de los diferentes países.

Intermediarios: Son las Asociaciones y los minoristas, estos últimos son los comercializadores detallistas y grandes superficies, en donde se agrupan los diferentes canales de distribución que facilitan el acceso directo del producto al consumidor internacional (Mosquera Abadía et al., 2016). Se debe aclarar que el principal mercado del aguacate Hass es a nivel internacional. Por este motivo, no se incluyeron estos intermediarios en este estudio. Por otro lado, las asociaciones, son la representación de los pequeños productores, que requieren representatividad para alcanzar negociaciones favorables, en muchos casos deben unirse para alcanzar niveles de producción y cumplir los requerimientos de volumen de las líneas de empaque.

Transporte primario y secundario: Se considera como un eslabón de la cadena, dado que las actividades desarrolladas son susceptibles a riesgos operacionales. El transporte primario se define como el transporte desde el productor de aguacate hasta la línea de empaque y el transporte secundario desde la línea de empaque hasta el puerto. Los actores en el secundario son mucho más especializados.

Consumidor final: Reúne a los individuos que demandan la fruta en fresco, tanto en el orden nacional como internacional (Mosquera Abadía et al., 2016).

Se debe tener en cuenta que dentro de la investigación realizada se consideran los eslabones de productor, línea de empaque, transporte primario y secundario debido principalmente a que en la revisión de literatura se encontró que los riesgos operacionales se presentan especialmente en estos y representan a gran parte de los actores de la cadena productiva.

\section{Mapa de cadena de valor de productor de aguacate}

Los procesos que se llevan a cabo en el eslabón productor inician con la entrega de productos desde los proveedores, pasan a la etapa de siembra, monitoreo, etapa en la que crece la planta, posteriormente a cosecha, preselección, almacenamiento y despacho del aguacate hacia la línea de empaque. En todas estas etapas se identificaron desperdicios o interrupciones en las actividades. Además, se representa una línea de tiempo en la que se identifican cuándo se producen pérdidas del producto, por ejemplo, las etapas de siembra, monitoreo del cultivo y cosecha, son etapas que agregan valor, pero contienen también actividades que no agregan valor y los resultados se evidencian posteriormente en la etapa de preselección, donde se hacen visibles las pérdidas del producto. Igualmente, en las etapas de almacenamiento y despacho, se presentan actividades que producen pérdidas por manipulación.

\section{Mapa de cadena de valor de transporte primario}

Considerando que el transporte es fundamental y generador de riesgos operacionales (Osorio et al., 2017), impacta el desempeño de la cadena productiva del aguacate por la naturaleza de las operaciones logísticas, se analiza el transporte primario con ayuda del VSM inicia desde que la línea de empaque o el productor contactan al transportador para recoger el producto en las fincas y llevarlo a la línea de empaque, el control de todas las operaciones del transporte se ejerce por alguno de los dos, según quien haya sido encargado de contratar el transporte. El transportador cuenta con camiones, el cual se programa para llegar al sitio, este debe garantizar algunas condiciones del vehículo; cuando llega al sitio se realiza el cargue del producto, se transporta el aguacate en canastillas hasta la línea de empaque, llega a la planta, una vez se verifican las condiciones se descarga. Se presentan desperdicios relacionados con los procesos o con manipulación, los cuales representan actividades que no agregan valor.

\section{Mapa de cadena de valor de línea de empaque}

En la planta de línea de empaque de aguacate Hass los procesos inician con la recepción del aguacate que viene desde los productores en camiones (ver Figura 3), pasan por limpieza y desinfección, lavado y sellado 
de pedúnculo, transporte hasta el secado, posteriormente clasificación según calibres, selección y empaque, rotulación, paleteado, almacenamiento en cuarto frío y cargue y despacho hacia el puerto marítimo por donde será enviado al cliente internacional. EI VSM de la figura 3 muestra los desperdicios presentados en cada una de las etapas y la representación de las actividades que no agregan valor.

Mapa de cadena de valor de transporte secundario

En el mapeo del transporte secundario, el proveedor es representado por la línea de empaque, donde está almacenado el producto empacado que será enviado a clientes internacionales y debe ser transportado vía terrestre hasta el puerto marítimo. El control es ejercido por la empresa de transporte contratada por la línea de empaque o por terceros que enviaron los productos a empacar y son quienes pudieron realizar las negociaciones de venta con los clientes internacionales. En cualquier caso, la línea de empaque informa a la empresa de transporte para programar la recogida del producto; el vehículo refrigerado llega a las instalaciones de la empacadora, en ese momento verifican la información del vehículo y del transportador, para garantizar que no se presenten irregularidades, puesto que existe un riesgo bastante alto de presencia de narcotráfico en la carga que se envíe.

\section{Fase 2: Identificación de los Riesgos}

Se identificaron 45 riesgos para el eslabón productor, 31 de ellos son de tipo biológico. Para el eslabón de transporte primario y secundario, se encontraron un total de 25 de ellos, donde los riesgos de tipo entrega y los mecánicos son los que más representativos con un total de 10 y 6 respectivamente. Y para el eslabón de línea de empaque se encontraron un total de 48 riesgos, destacando que los asociados a la ausencia de procesos y el riesgo de fallo en la infraestructura, instalaciones y/o máquinas son los más destacados (ver tabla 1).

Tabla 1: Riesgos identificados en los eslabones de la cadena de suministro del Aguacate Hass.

\begin{tabular}{|c|c|c|}
\hline Eslabón & Tipología de Riesgos & No. Riesgos \\
\hline \multirow{4}{*}{ Productor } & Biológicos & 31 \\
\hline & Mecánicos & 4 \\
\hline & Abastecimiento & 6 \\
\hline & Ambientales & 4 \\
\hline \multirow{4}{*}{ Transporte primario/secundario } & Físicos/químicos & 5 \\
\hline & Sociales, económicos y ambientales & 4 \\
\hline & Mecánicos & 6 \\
\hline & Entrega & 10 \\
\hline \multirow{8}{*}{ Línea de empaque } & Mecánicos & 2 \\
\hline & Fallo infraestructura/equipos & 8 \\
\hline & Riesgos procesos & 14 \\
\hline & Calidad & 5 \\
\hline & Almacenamiento & 3 \\
\hline & Sociales, económicos y ambientales & 2 \\
\hline & Físicos/químicos & 5 \\
\hline & Entrega & 9 \\
\hline
\end{tabular}




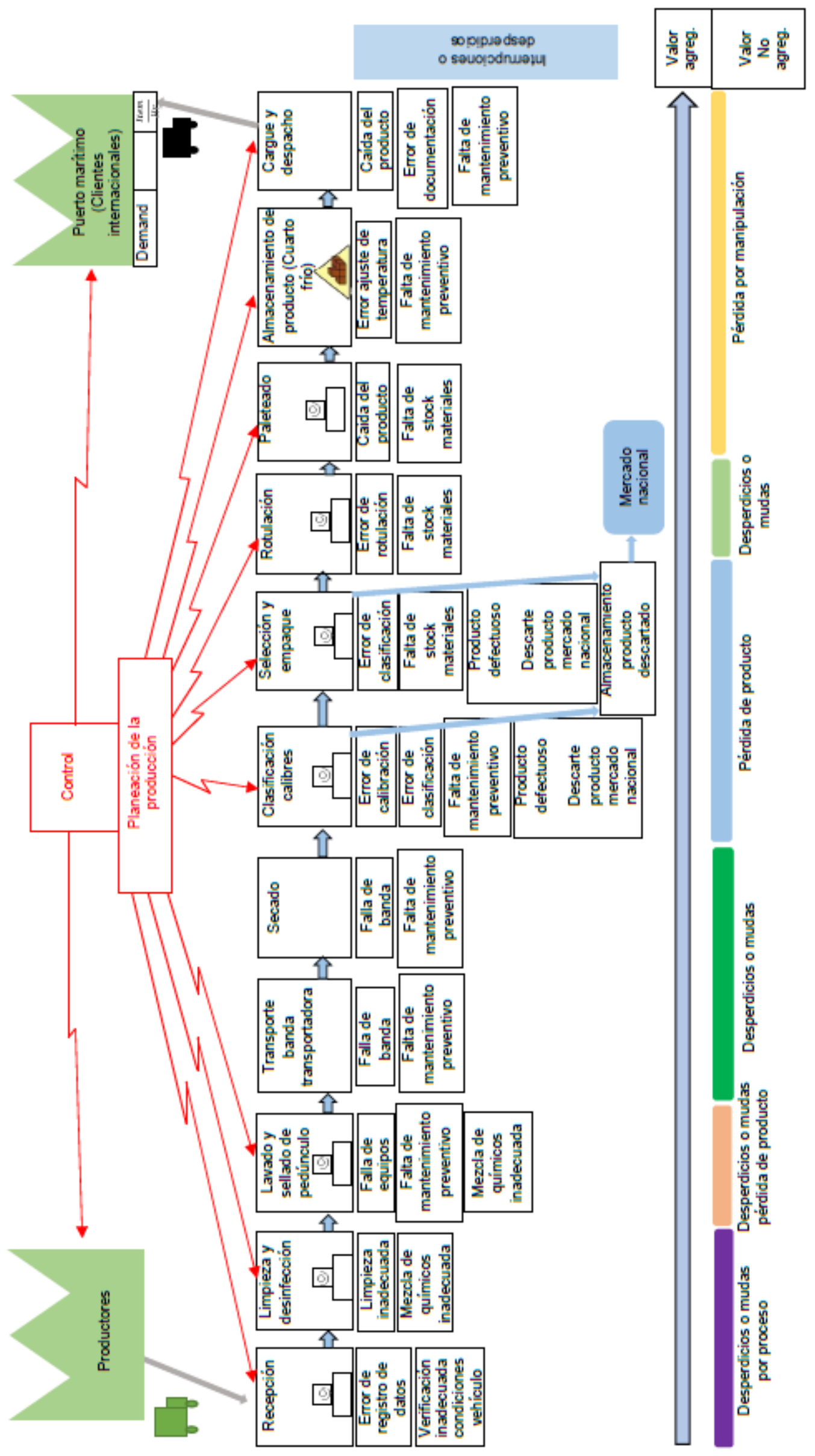

Fig. 3: Mapa de cadena de valor actual para el eslabón línea empaque de la cadena de Aguacate Hass. 


\section{Fase 3: Priorización de los riesgos}

Se priorizaron aquellos riesgos críticos para la operación de cada eslabón y se consolidaron en sus categorías principales. Luego de aplicar el método AMFE a los riesgos definidos por cada eslabón, se procede a seleccionar los 8 (Tabla 2) con un mayor índice de priorización final. Después se realizó una priorización de los riesgos operacionales de cada eslabón teniendo en cuenta el juicio de expertos dentro de cada uno de los agentes de la cadena con la herramienta QFD difuso. Así se conoce cuál es el riesgo más prioritario y se sabe, a partir de cuáles riesgos se considera que el impacto sobre la organización es alto, con el objeto de definir las acciones y medidas a implementar. Para la aplicación del QFD difuso se desarrolló una herramienta en Excel, aplicando una a una las etapas propuestas por Osorio et al. (2018), con lo cual se logró obtener un valor de Indicador de Prioridad de Riesgo Final (IPRF) por cada riesgo en cada eslabón.

Tabla 2: Riesgos priorizados en los eslabones de la cadena de suministro del Aguacate Hass

\begin{tabular}{|c|c|c|c|c|c|}
\hline$E$ & Riesgo & IPRF & $E$ & Riesgo & IPRF \\
\hline \multirow{8}{*}{$\begin{array}{l}\frac{\overline{0}}{0} \\
\frac{0}{0} \\
\frac{0}{0} \\
\frac{0}{2}\end{array}$} & $\begin{array}{l}\text { Riesgo de insecto Pasador de fruto - } \\
\text { pasador de ramas }\end{array}$ & 468 & \multirow{8}{*}{ 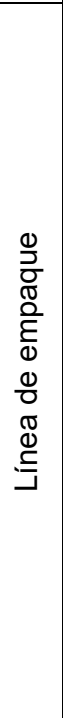 } & $\begin{array}{l}\text { Riesgo de que el motor del cuarto frío no } \\
\text { funcione de manera adecuada }\end{array}$ & 549 \\
\hline & $\begin{array}{l}\text { Riesgo de insecto Barrenador de la } \\
\text { semilla - (Picudo). }\end{array}$ & 444 & & Límites máximos y mínimos de temperatura & 540 \\
\hline & $\begin{array}{l}\text { Riesgo de insecto Monalonion } \\
\text { velezangieli - Monalonion dissimulatum }\end{array}$ & 431 & & Pedidos retrasados & 525 \\
\hline & $\begin{array}{l}\text { Riesgo de insecto Phylophaga Persea } \\
\text { - Cucarron marseño }\end{array}$ & 382 & & $\begin{array}{l}\text { Límites máximos y mínimos químicos en el } \\
\text { tratamiento (Residualidad) }\end{array}$ & 452 \\
\hline & Riesgo de insecto Barrenador de tallo & 333 & & Utilización de personal innecesario & 442 \\
\hline & $\begin{array}{l}\text { Riesgo de insectoc complejo ácaros } \\
\text { Polyphagotarsonemus latus- } \\
\text { Olygonichus yothersii - Tetranychus } \\
\text { urticae }\end{array}$ & 333 & & Altos niveles de estrés & 308 \\
\hline & $\begin{array}{l}\text { Riesgo de insecto COMPLEJO } \\
\text { THRIPS, Frankiniella occidentalis, } \\
\text { Heliothrips haemorrhoidalis. }\end{array}$ & 321 & & Caída de las estibas, canastillas o frutas & 292 \\
\hline & $\begin{array}{l}\text { Riesgo de enfermedad Muerte } \\
\text { descendente }\end{array}$ & 271 & & Riesgo de fallo en la máquina calibradora & 277 \\
\hline$E$ & Riesgo & IPRF & $E$ & Riesgo & IPRF \\
\hline \multirow{8}{*}{ 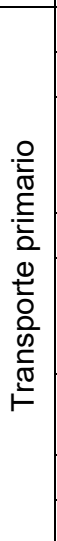 } & Fallas técnicas de los vehículos & 181 & \multirow{8}{*}{ 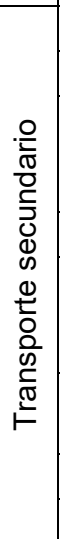 } & Riesgo por contaminación de narcotráfico & 541 \\
\hline & Flota inapropiada & 177 & & Riesgo por rupturas en la cadena de frío & 541 \\
\hline & $\begin{array}{l}\text { Prácticas, condiciones o equipos } \\
\text { inapropiados para el cargue y } \\
\text { descargue }\end{array}$ & 166 & & Riesgo de volcamiento & 505 \\
\hline & Vías en mal estado & 157 & & Riesgo por fallas técnicas en los vehículos & 487 \\
\hline & $\begin{array}{l}\text { Daños Mecánicos o lesiones al } \\
\text { aguacate (Cortes, Rasguños, impactos } \\
\text { y compresión) }\end{array}$ & 154 & & $\begin{array}{l}\text { Riesgo de caídas del aguacate dentro del } \\
\text { vehículo }\end{array}$ & 419 \\
\hline & Contaminación cruzada & 132 & & $\begin{array}{l}\text { Riesgo por Demoras por inspecciones de las } \\
\text { autoridades }\end{array}$ & 255 \\
\hline & Contaminación biológica & 128 & & Riesgo por paros, huelgas, manifestaciones & 199 \\
\hline & Huelgas, paros, manifestaciones & 127 & & Riesgo por Desastres en las vías & 199 \\
\hline
\end{tabular}

\section{Fase 4: Mitigación de los Riesgos}

Con el fin de establecer acciones de mitigación que sean transversales, es decir, que impacten varios riesgos de la misma categoría, se desarrolla la matriz de relación riesgos, desperdicios y estrategias de mitigación (Tabla 3), como se puede observar relaciona los riesgos por eslabón y los desperdicios (defectos, esperas, movimientos y sobreprocesamiento), con lo cual se pueden establecer acciones de mitigación según la aplicación de algunas herramientas de manufactura esbelta como lo son TPM (Mantenimiento productivo total, por sus siglas en inglés), 5s, Gestión visual y Estandarización, encontrando en estas una pertinencia de aplicación para mitigar los riesgos y/o desperdicios identificados en los respectivos VSM actuales de cada eslabón. En este análisis se puede identificar que en todos los eslabones se presentan defectos, y es el desperdicio que más se presenta, seguido de esperas, movimientos y sobreprocesamiento, además que para dichos desperdicios y los riesgos, pueden mitigarse principalmente con Estandarización de los procesos, Implementación de Gestión visual, TPM y 5s. Teniendo identificados los riesgos priorizados en los cuatro eslabones de la cadena de suministro, la relación con los desperdicios y a su vez con las herramientas de manufactura esbelta, se procede a identificar acciones de mitigación que permitan reducir y/o eliminar el 
efecto que pueden provocar la ocurrencia de estos riesgos en los procesos operacionales de las compañías asociadas a la cadena productiva del aguacate.

Tabla 3: Matriz de relación de los riesgos de la cadena productiva del aguacate, desperdicios y acciones de mitigación con un enfoque de manufactura esbelta

\begin{tabular}{|c|c|c|c|c|c|c|c|c|c|}
\hline \multicolumn{2}{|c|}{$\begin{array}{l}\text { Análisis de acciones de mitigación en la Cadena productiva del } \\
\text { aguacate Hass }\end{array}$} & \multicolumn{4}{|c|}{$\begin{array}{c}\text { Desperdicios enfoque } \\
\text { de manufactura } \\
\text { esbelta }\end{array}$} & \multicolumn{4}{|c|}{$\begin{array}{l}\text { Acciones de } \\
\text { mitigación }\end{array}$} \\
\hline Eslabón & Riesgos & 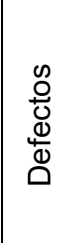 & $\begin{array}{l}\frac{0}{\pi} \\
\frac{\pi}{0} \\
\frac{0}{0} \\
\ddot{w}\end{array}$ & 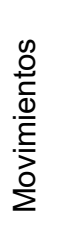 & 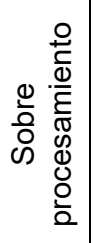 & $\sum_{\substack{1 \\
F}}$ & $\stackrel{\sim}{\sim}$ & 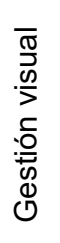 & 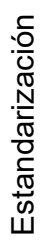 \\
\hline Productor & Biológicos & $\mathrm{X}$ & $\mathrm{X}$ & $\mathrm{X}$ & $\mathrm{X}$ & $\mathrm{X}$ & $\mathrm{X}$ & $\mathrm{X}$ & $x$ \\
\hline \multirow{5}{*}{$\begin{array}{l}\text { Transporte } \\
\text { primario }\end{array}$} & Riesgo por Fallas en los equipos & & $\mathrm{X}$ & & & $\mathrm{X}$ & & & $\mathrm{X}$ \\
\hline & Riesgo por flota inapropiada & $x$ & $x$ & & $\mathrm{x}$ & & & $x$ & $x$ \\
\hline & Riesgos mecánicos & $\mathrm{X}$ & $\mathrm{x}$ & $x$ & & $x$ & & & $\mathrm{X}$ \\
\hline & Riesgos por contaminación cruzada & $\mathrm{X}$ & & & $x$ & $\mathrm{X}$ & $x$ & $x$ & $x$ \\
\hline & Riesgo por contaminación biológica & $\mathrm{X}$ & & & $\mathrm{X}$ & $\mathrm{X}$ & $x$ & $\mathrm{X}$ & $x$ \\
\hline \multirow{3}{*}{$\begin{array}{l}\text { Línea de } \\
\text { empaque }\end{array}$} & Riesgos Mecánicos & $\mathrm{x}$ & & $x$ & & $x$ & $x$ & $x$ & $x$ \\
\hline & Riesgos fallas equipos & $x$ & $x$ & & & $\mathrm{x}$ & $x$ & $x$ & $x$ \\
\hline & Riesgos procesos & $x$ & $\mathrm{x}$ & $x$ & & & $x$ & $x$ & $\mathrm{X}$ \\
\hline \multirow{4}{*}{$\begin{array}{l}\text { Transporte } \\
\text { secundario }\end{array}$} & Riesgo contaminación de narcotráfico & $\mathrm{x}$ & & & & & & $x$ & $\mathrm{X}$ \\
\hline & Riesgo ruptura en la cadena de frío & $x$ & & & & $x$ & & $x$ & $x$ \\
\hline & Riesgos fallas equipos (vehículos) & & $x$ & $x$ & & $x$ & $x$ & $x$ & $\mathrm{X}$ \\
\hline & Riesgos mecánicos (caída del aguacate) & $X$ & $\mathrm{X}$ & & & & & $\mathrm{X}$ & $x$ \\
\hline
\end{tabular}

\section{Acciones de mitigación para el eslabón productor}

Los riesgos con un mayor impacto sobre la operación de los productores radican principalmente en plagas de distintos insectos que pueden provocar grandes pérdidas económicas para este eslabón. Según (ICA, 2012) la causa principal de enfermedades y plagas en los cultivos se debe a un inadecuado manejo del cultivo, el cual incluye actividades como falta de fumigación, ausencia de podas sanitarias y deficientes prácticas y/o conocimientos de los operarios que trabajan en el campo, lo que refleja la ausencia de un plan de monitoreo al cultivo. A partir de este análisis, la primera estrategia de mitigación que se plantea es la ejecución de un plan de orden y aseo de las herramientas utilizadas por los operarios para el corte de producto basado en la técnica de manufactura esbelta 5S, la cual ayuda a disminuir las actividades que no agregan valor, incrementando la productividad en las empresas y mejorando la calidad del producto (Omogbai y Salonitis, 2017), este último indicador siendo de un gran interés para la cadena productiva del aguacate, donde el no tener un producto de alta calidad impide la exportación del mismo y genera detrimentos en el flujo de efectivo proveniente de su operación.

Como segunda estrategia de mitigación se plantea la definición de un plan de mantenimiento del cultivo basado en la técnica de manufactura esbelta, Mantenimiento Productivo Total (TPM) debido principalmente a que permite establecer un control a planes de mantenimiento, con lo cual elimina pérdidas en los procesos, reduce el tiempo de inactividad y minimiza costos (Pinto et al., 2020). Caicedo-Solano et al. (2020) proponen un modelo matemático como herramienta de apoyo a la toma de decisiones para problemas de planificación del mantenimiento de cultivos de fincas bananeras en Colombia. El modelo busca reducir los desperdicios asociados a manufactura esbelta que incluye mano de obra, uso de maquinaria y operaciones en ventanas de tiempo que preservan la calidad de la cosecha. Por el contrario, en este artículo se plantea el TPM asociado a actividades de mantenimiento autónomo y entrenamiento para garantizar un correcto control del cultivo de aguacate Hass, lo cual fomentaría la polivalencia y conocimiento de los empleados a la hora de identificar si el cultivo está siendo atacado por una plaga, lo que conllevaría a una respuesta temprana y por ende provocaría la reducción de posibles pérdidas de producto causadas por la enfermedad. 
Como complemento a la implementación del TPM, se plantea como tercera estrategia de mitigación, la estandarización (Standard Work) del proceso de mantenimiento del cultivo como una estrategia necesaria para documentar el proceso correcto, establecer indicadores de seguimiento y facilitar el trabajo de los empleados. Es necesario tener en cuenta que la técnica de trabajo estandarizado conlleva a la mejora de la calidad de los procesos, al establecer un procedimiento fácil de comunicar y que puede ser aplicado por cualquier empleado de la organización (O'Reilly et al., 2016). Asimismo Reis et al. (2018) reconocen como la estandarización de procesos a través de la documentación de la información y la medición de indicadores son esenciales para determinar la madurez, en términos de manufactura esbelta, para este sector. Barth y Melin (2018) describen cómo el uso de las sesiones de capacitación, las visitas a las fincas, los talleres y el asesoramiento para presentar a los agricultores los beneficios y riesgos de la implementación de un modelo de manufactura esbelta, reduce los desperdicios en el proceso, aumentando la producción y los ingresos.

La cuarta estrategia de mitigación que se plantea es la implementación de una metodología para la gestión visual de los cultivos, el cual permite crear un lenguaje común y sencillo de entender entre todos los operarios de una organización, con el propósito de garantizar su control y seguimiento (Singh y Kumar, 2020). Esta estrategia sería un complemento para TPM y el trabajo estandarizado, ya que, en muchas ocasiones los procedimientos establecidos no logran ser comunicados efectivamente a los empleados, lo que dificulta la aplicación de estos. De esta manera, se representó un estado futuro para el productor, donde se propone la implementación de las herramientas de manufactura esbelta antes mencionadas, según los estallidos de eventos Kaizen, con lo cual se lograría eliminar los desperdicios y obtener unas mejoras en los procesos, lo que se traduce en la mitigación de los riesgos operacionales, y no solamente los priorizados, como los biológicos sino riesgos de calidad, de fallas en los equipos de procesos y riesgos mecánicos.

\section{Acciones de mitigación para el eslabón línea de empaque}

Los riesgos más relevantes en las líneas de empaque se encuentran concentrados en operaciones como mantenimiento de cadena de frío, tratamientos químicos, transporte y manipulación del aguacate, proceso de calibración y entrega de producto (para ver con detalle cada uno de los riesgos, véase la tabla 1). En el caso de la caída de estibas, canastilla y frutas se propone hacer seguimiento al indicador de averías en términos de días, semanas o meses y que constituya una herramienta para los trabajadores y para supervisores, hacia la disminución de este riesgo. Del mismo modo, se plantea aplicar un programa de $5 \mathrm{~S}$ en el área de almacén de insumos, lo que permite llevar un control exhaustivo de estos materiales para evitar desabastecimiento que generen retrasos en la línea de empaque.

Se propone revisar los indicadores de la integración entre los sistemas esbeltos y verdes propuesta por Reis et al. (2018) para reducir los riesgos de temperatura y residualidad de químicos en el tratamiento. El trabajo estándar evita la ejecución de estas tareas de forma aleatoria. Asimismo, es necesario implementar programas de mantenimiento productivo que fomenten la autonomía en el personal, actividades que eviten averías de las máquinas que afecten la calidad del producto y el incremento en los costos de proceso. De acuerdo con los autores arriba citados, existen varios niveles de madurez en la integración; uno de los cuales es la estandarización. En este nivel los sistemas pueden coexistir, aunque no estén integrados, los objetivos no estén cuantificados y se aplican indicadores específicos. Esta integración contribuye a los aspectos de sostenibilidad en los diferentes actores de la cadena de suministro del aguacate Hass.

Riesgos como utilización de personal innecesario y altos niveles de estrés se pueden abordar desde la planeación, la cual constituye una acción para mitigar los altos niveles de estrés ya que pueden conducir a defectos. La utilización de personal innecesario se relaciona con sobreprocesamiento y subutilización del talento humano. La planeación mejorada puede apoyarse en modelos que integren la reducción de desperdicios de manufactura esbelta como lo proponen Caicedo-Solano et al. (2020). Estos autores desarrollaron un modelo matemático para minimizar los costos de producción e incluye la reducción de desperdicios como esperas y calidad. En este sentido, la implementación de dichas acciones de mitigación puede representar la eliminación de desperdicios y mitigación o eliminación de riesgos operacionales. En la figura 4 se presenta el Mapa futuro del eslabón línea de empaque, donde se representan las estrategias de mitigación asociadas a las herramientas de manufactura esbelta como estallidos Kaizen y al mismo tiempo las acciones de mitigación asociadas a dichas herramientas, y los riesgos operacionales que se impactarían. Es evidente que estas estrategias no solamente impactan los riesgos que fueron priorizados sino otros riesgos presentes en la cadena, reflejando un beneficio significativo para el eslabón y para la cadena, principalmente en la eliminación de producto defectuoso.

\section{Acciones de mitigación para eslabón transporte primario y secundario}

Teniendo en cuenta los riesgos presentados en la tabla 1, se puede observar cómo los riesgos se concentran principalmente en los procedimientos asociados a la entrega, la ausencia de un plan de mantenimiento preventivo para los vehículos, las malas prácticas de cargue y descargue y por último causas asociadas a 
detrimentos en el estado de las carreteras, inspecciones por autoridades y manifestaciones sociales de la población.

Se propone la herramienta de trabajo estándar para mitigar los riesgos de caídas y deterioro del aguacate durante el transporte. El trabajo estándar es un método que define cómo se deben desarrollar las actividades en un puesto de trabajo, previniendo así que el operador las ejecute aleatoriamente (Bragança y Costa, 2015). En este caso se recomienda usar tablas de capacidad de partes y producción, gráficas de combinación de operaciones estándar y hoja de trabajo estándar; todas ellas definidas en la herramienta de trabajo estándar. Con el uso de esta herramienta se reduce la variabilidad y se mejora la calidad.

Similarmente el trabajo estándar se propone para mitigar las rupturas de cadena de frío al contar con procedimientos estrictos y formales que eviten la aleatoriedad. Estos eventos dan lugar a la aparición de defectos que se pueden mitigar con la integración de los sistemas de manufactura esbelta (Barth y Melin (2018); Reis et al. (2018)). Las $5 S$ pueden intervenir también así como la gestión visual, en las rupturas de la cadena de frío ya que son herramientas sencillas que se reportan como las más usadas entre granjeros y agricultores para manejar rutinas de trabajo y estandarizar la ubicación de herramientas y equipos de trabajo (Barth y Melin, 2018). Por último, se plantea la generación de un programa de mantenimiento productivo que permita minimizar las paradas de los vehículos por fallas técnicas, al mismo tiempo que se garantiza las condiciones óptimas para el transporte del producto.

Según el VSM futuro del eslabón transporte primario, se relacionan las estrategias y las acciones de mitigación que eliminan o mitigan los desperdicios y los riesgos operacionales con acciones propuestas, como se puede observar, además de los riesgos priorizados también se ven impactados otros riesgos, con esto se espera que las actividades que no agregan valor desaparezcan y solo se mantenga aquellas que agregan valor. Asimismo, de acuerdo con el VSM futuro del eslabón transporte secundario, las estrategias de mitigación asociadas a las herramientas de manufactura esbelta tales como 5S, TPM, Estandarización o trabajo estándar y Gestión visual. Además, se puede observar las acciones de mitigación propuestas para reducir o eliminar los riesgos operacionales en el transporte secundario, que, si bien se realiza de manera organizada, liderada por empresas formales y estructuradas, es importante que se garantice que la presencia de los riesgos es baja o el impacto también será bajo en caso de que se dé.

\section{CONCLUSIONES}

La cadena productiva del aguacate Hass en el Valle del Cauca - Colombia está compuesta por productores, transporte primario, línea de empaque y transporte secundario, eslabones fundamentales en la operación de la cadena de exportación del producto y que representan, por tanto, la fuente principal de riesgos operacionales que pueden impactar las actividades normales de cada eslabón en la cadena. Aunque se evidencia que existe una mayor cantidad de productores para satisfacer la demanda, quien lidera o coordina es la línea de empaque, puesto que esta planta es la que conoce los requerimientos de los clientes internacionales y tiene puede verificar y controlar la calidad y las especificaciones del producto.

Se identificaron los principales riesgos operacionales en la cadena productiva del aguacate Hass para cada uno de los eslabones, es decir, aquellos que presentan mayor probabilidad de ocurrencia e impacto a los objetivos estratégicos, reconociendo como principales los riesgos biológicos como el riesgo por insectos y enfermedades en el productor; los riesgos mecánicos como el de fallas técnicas en los vehículos en el transporte primario; y los de fallos en infraestructura como el de que el motor del cuarto frío no funcione adecuadamente en la línea de empaque; y el de contaminación de narcotráfico en el transporte secundario.

Se seleccionaron los riesgos de mayor impacto en cada uno de los eslabones por medio de QFD difuso, con lo cual se logra obtener un marco de referencia para la gestión de los mismos, mediante la participación de los stakeholders, obteniendo la percepción sobre el impacto en los objetivos estratégicos y los deseos que se espera obtener para cada eslabón, con el fin de proponer acciones de mitigación encaminadas a mitigar los principales riesgos operacionales.

Se propusieron estrategias de mitigación de los riesgos priorizados con base en la filosofía de manufactura esbelta, basada en las herramientas TPM, 5S, Estandarización y Gestión visual. El detalle o ajuste de cada acción se recomienda ejecutarlo en conjunto con el personal de las empresas de cada eslabón para revisar sus particularidades y sacar el mayor beneficio de las propuestas. Las propuestas de herramientas de manufactura esbelta se definieron con la herramienta VSM o mapeo de la cadena de valor, encontrando una relación entre riesgos y desperdicios, para proponer estrategias basadas en TPM, 5s, Gestión visual y Estandarización para mitigar los riesgos. 


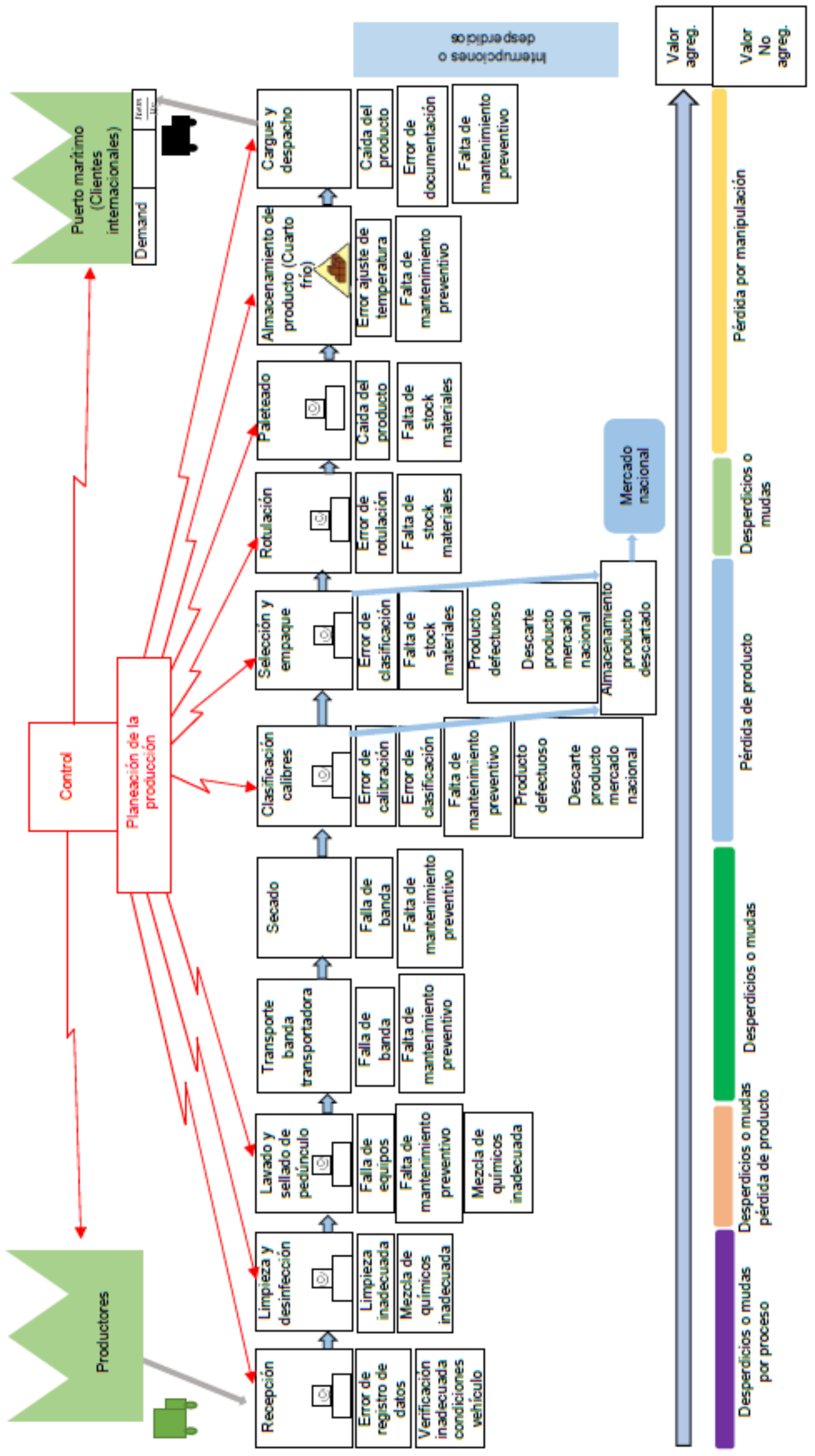

Fig. 4: Mapa de cadena de valor futuro para el eslabón línea empaque de la cadena Aguacate Hass 


\section{REFERENCIAS}

Barth, H., y Melin, M. A., Green Lean approach to Global Competition and Climate Change in the Agricultural Sector - A Swedish Case Study, https://doi.org/10.1016/j.jclepro.2018.09.021, Journal of Cleaner Production, 204, 183-192 (2018).

Blome, C., Schoenherr, T., y Eckstein, D., The Impact of knowledge Transfer and Complexity on Supply Chain Flexibility: A knowledge-based View, https://doi.org/10.1016/j.ijpe.2013.02.028, International Journal of Production Economics, 147, 307-316 (2014).

Bragança, S., y Costa, E., An application of the Lean Production Tool Standard Work, https://doi.org/10.11113/jt.v76.3659, Jurnal Teknologi, 76(1), 47-53 (2015).

Caicedo-Solano, N. E., García-Llinás, G. A., Montoya-Torres, J. R., y Ramirez-Polo, L. E., A Planning Model of Crop Maintenance Operations Inspired in Lean Manufacturing, https://doi.org/10.1016/j.compag.2020.105852, Computers and Electronics in Agriculture, 179, 105852 (2020).

Cardozo, E. R., Rodríguez, C., y Guaita, W., Las Pequeñas y Medianas Empresas Agroalimentarias en Venezuela y el Desarrollo Sustentable: Enfoque basado en los Principios de Manufactura Esbelta, https://doi.org/10.4067/S071807642011000500006, Información Tecnológica, 22(5), 39-48 (2011).

CRC, Narrativa de Competitividad del Valle del Cauca. Las 5 fortalezas del Valle del Cauca para alcanzar un futuro extraordinario, Comisión Regional de Competitividad Del Valle Del Cauca, https://www.viveelritmodelpacifico.co/wpcontent/uploads/2017/12/CRC-Narrativa-Un-Valle-que-se-Atreve-V3.pdf (2015).

De Steur, H., Wesana, J., y otros tres autores, Applying Value Stream Mapping to Reduce Food Losses and Wastes in Supply Chains: A Systematic Review, https://doi.org/10.1016/j.wasman.2016.08.025, Waste Management, 58, 359-368 (2016).

Delgado-Álvarez, N., Covas-Varela, D., y Martínez-Curbelo, G., Aplicación del mapa de flujo de valor (Value Stream Map-Vsm) a La Gestión De Cadenas De Suministros De Productos Agrícolas: Un Caso De Estudio, https://doi.org/10.37611/ib2ol1119-135, Identidad Bolivariana, 2(1), 119-135 (2018).

Escobar, J. V., Rodriguez, P., Cortes, M., y Correa, G., Influencia de la Materia Seca como Índice de Madurez de Cosecha y Tiempo de Almacenamiento en Frío sobre la Calidad del Aguacate cv. Hass Producido en la Región del Trópico Alto, https://doi.org/10.4067/s0718-07642019000300199, Información Tecnológica, 30(3), 199-210 (2019).

ICA, Manejo fitosanitario del cultivo del Aguacate Hass, Instituto Colombiano Agropecuario, https://www.ica.gov.co/getattachment/4b5b9b6f-ecfc-46e1-b9ca-b35cc1cefee2/- (2012).

Kırılmaz, O., y Erol, S., A Proactive Approach to Supply Chain Risk Management: Shifting Orders Among Suppliers to Mitigate the Supply Side Risks, Journal of Purchasing and Supply Management, 23(1), 54-65 (2017).

Lavastre, O., Gunasekaran, A., y Spalanzani, A., Supply Chain Management in French Companies, https://doi.org/10.1016/j.dss.2011.11.017, Decision Support Systems, 52(4), 828-838 (2012)

Méndez, M. C., Estrategias competitivas del Eslabón Primario en la Cadena Productiva de Aguacate de los Montes de María, https://doi.org/10.1016/j.pursup.2016.04.002, Revista de Economía y Administración, 13, 95-110 (2016).

Mosquera, H. A., Toro, J. C., y Betancourt, B., Estudio Prospectivo de la Cadena Productiva de la Pitaya Amarilla en Fresco en el Valle del Cauca, Primera Edición, 1-200, Programa Editorial Universidad del Valle, Santiago de Cali, Colombia, (2016).

Nakandala, D., Lau, H., y Zhao, L., Development of a Hybrid Fresh Food Supply Chain Risk Assessment Model, https://doi.org/10.1080/00207543.2016.1267413, International Journal of Production Research, 55 (14), $4180-4195$ (2016).

Narasimhan, R., y Talluri, S., Perspectives on Risk Management in Supply Chains, https://doi.org/10.1016/j.jom.2009.02.001, Journal of Operations Management, 27(2), 114-118 (2009).

O'Reilly, K., Ruokis, S., y otros seis autores, Standard Work for Room Entry: Linking Lean, Hand Hygiene, and Patientcenteredness, https://doi.org/10.1016/j.hjdsi.2015.12.008, Healthcare, 4(1), 45-51 (2016).

Omogbai, O., y Salonitis, K., The Implementation of 5S Lean Tool Using System Dynamics Approach, https://doi.org/10.1016/j.procir.2017.01.057, Procedia CIRP, 60, 380-385 (2017).

Osorio, J. C., Manotas, D. F., y Rivera, L., Priorización de Riesgos Operacionales para un Proveedor de Tercera Parte Logística - 3PL, https://doi.org/10.4067/S0718-07642017000400016, Información Tecnológica, 28(4), 135-144 (2017).

Osorio, J. C., Manotas, D. F., Rivera, L., y Canales, I., Operational Risk Prioritization in Supply Chain with 3PL Using Fuzzy-QFD, In New Perspectives on Applied Industrial Tools and Techniques, Springer, ISBN: 978-3-319-56871-3, 91109, https://doi.org/10.1007/978-3-319-56871-3, 91-109, Springer, Cham. Switzerland, (2018).

Pinto, G., Silva, F. J. G., y otros cuatro autores, TPM Implementation and Maintenance Strategic Plan - a Case Study, https://doi.org/10.1016/j.promfg.2020.10.198, Procedia Manufacturing, 51, 1423-1430 (2020).

Prakash, S., Soni, G., y otros tres autores, Risk Analysis and Mitigation for Perishable Food Supply Chain: A Case of Dairy Industry, https://doi.org/10.1108/IJEBR-02-2016-0064, Benchmarking: An International Journal, 24(1), 2-23 (2017). 
Reis, L. V., Kipper, L. M., y otros cinco autores, A Model for Lean and Green Integration and Monitoring for the Coffee Sector, https://doi.org/10.1016/j.compag.2018.03.034, Computers and Electronics in Agriculture, 150, 62-73 (2018)

Rivera-Cadavid, L., Justificación Conceptual de un Modelo de Implementación de Lean Manufacturing, Heurística, ISSN: 2422-5177,15, 91-106 (2011).

Singh, S., y Kumar, K., A Study of Lean Construction and Visual Management Tools Through Cluster Analysis, https://doi.org/10.1016/j.asej.2020.04.019, Ain Shams Engineering Journal, 12(1), 1153-1162 (2020).

Sodhi, M. S., Son, B. G., y Tang, C. S., Researchers' Perspectives on Supply Chain Risk Management, https://doi.org/10.1111/j.1937-5956.2011.01251.x, Production and Operations Management, 21(1), 1-13 (2012).

Tang, C. S., Perspectives in Supply Chain Risk Management, https://doi.org/10.1016/j.ijpe.2005.12.006, International Journal of Production Economics, 103(2), 451-488 (2006).

Venkatesh, V. G., Rathi, S., y Patwa, S., Analysis on Supply Chain Risks in Indian Apparel Retail Chains and Proposal of Risk Prioritization Model using Interpretive Structural Modeling, https://doi.org/10.1016/j.jretconser.2015.06.001, Journal of Retailing and Consumer Services, 26, 153-167 (2015).

Wesana, J., Gellynck, X., y otros tres autores, Measuring food and nutritional losses through value stream mapping along the dairy value chain in Uganda, https://doi.org/10.1016/j.resconrec.2019.104416, Resources, Conservation and Recycling, 150, 104416 (2019).

Zhao, G., Liu, S., y otros cinco autores, Risk analysis of the agri-food supply chain: A multi-method approach, https://doi.org/10.1080/00207543.2020.1725684, International Journal of Production Research, 58(16), 4851-4876 (2020). 